\title{
Optimizing cerebral blood flow: Hitting the sweet spot on cardiopulmonary bypass
}

\author{
Derrick Y. Tam, MD, and Stephen E. Fremes, MD, MSc, FRCSC, FACP, FACC
}

\footnotetext{
From the Division of Cardiac Surgery, Schulich Heart Centre, Department of Surgery, Sunnybrook Health Sciences Centre, University of Toronto, Toronto, Ontario, Canada.

Disclosures: Authors have nothing to disclose with regard to commercial support.

Received for publication April 24, 2017; accepted for publication May 5, 2017; available ahead of print June 16, 2017.

Address for reprints: Stephen E. Fremes, MD, MSc, FRCSC, FACP, FACC, Schulich Heart Centre, Sunnybrook Health Sciences Centre, Room H4 05, 2075 Bayview Ave, Toronto, Ontario, Canada M4N 3M5 (E-mail: Stephen.fremes@sunnybrook.ca).

J Thorac Cardiovasc Surg 2017;154:1588-9

$0022-5223 / \$ 36.00$

Copyright (c 2017 by The American Association for Thoracic Surgery

http://dx.doi.org/10.1016/j.jtcvs.2017.05.032
}

Permanent postoperative neurologic changes remain some of the most-feared consequences associated with the use of cardiopulmonary bypass (CPB) during cardiac surgery. Temporary neurologic dysfunction (delirium) complicates many operations, is associated with prolonged length of stay, and predisposes to additional complications. Whereas low systemic perfusion resulting in cerebral hypoperfusion has been implicated in postoperative stroke, ${ }^{1}$ elevated mean arterial pressure (MAP) beyond the cerebral autoregulation zone is associated with postoperative delirium. ${ }^{2}$ As such, maintaining MAP in a range to achieve cerebral autoregulation is one of the objectives during CPB. ${ }^{3,4}$ The difficulty lies in identifying that specific blood pressure in an individual patient in real time.

In this issue of the Journal, Hori and colleagues ${ }^{5}$ address an important topic related to CPB physiology: the optimal MAP to achieve autoregulation of cerebral blood flow (CBF). The authors are to be commended on their dedication and commitment in examining such an important issue in contemporary cardiac surgery. Their results were derived from 3 prospective studies conducted between 2008 and 2015 that used transcranial Doppler (TCD) to measure $\mathrm{CBF}$ and then correlated these measurements with MAP. In brief, using a previously validated method, a moving Pearson correlation coefficient between changes in MAP and mean middle cerebral artery blood flow velocity was used to generate the mean velocity index (Mx). ${ }^{6,7}$ When Mx was $<0.4$, the MAP was within the range for autoregulation, whereas as $\mathrm{Mx}$ approached 1, the MAP was outside of this range-that is, cerebral blood flow is pressure-dependent. In this study, cutoffs for lower and upper limits of autoregulation also were defined by extension.

Previous work has demonstrated the clinical utility of real-time $\mathrm{CBF}$ monitoring with TCD in patients with traumatic brain injury in the intensive care unit setting in the management of MAP and outcome prognostication. ${ }^{6}$ Applying this sophisticated analysis to 614 patients

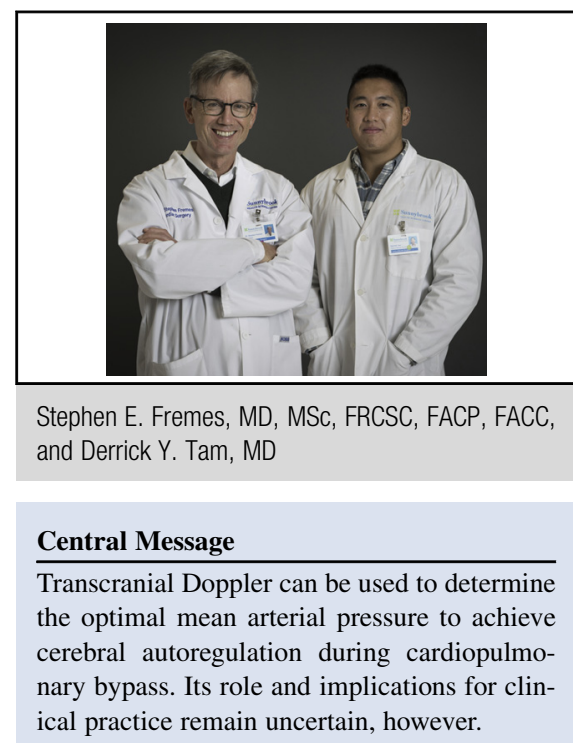

See Article page 1590. undergoing $\mathrm{CPB}$, the authors showed that the optimal MAP for maintaining cerebral autoregulation was $78 \pm 11 \mathrm{~mm} \mathrm{Hg}$ in approximately $70 \%$ of patients.

Nonetheless, this study has some limitations that warrant discussion. Whereas 614 patients were enrolled, more than 2000 patients were excluded from the study. Only patients at high risk for neurologic complications were included, and those who did not have a temporal window for TCD monitoring were excluded. Accordingly, the neurologic benefits may be more pronounced in the patients analyzed compared with the average-risk cardiac surgery population. Furthermore, the optimal MAP could be defined for only $54 \%$ of patients because $17 \%$ of patients were below the limit of autoregulation and $29 \%$ were above this limit. Given the foregoing limitations, the generalizability of such an approach to all patients undergoing cardiac surgery is reduced. Whereas a strength of this study includes monitoring for neurologic events as defined by the Society of Thoracic Surgeons' national database, the authors reported only the outcome of strokes, which occurred in only $5 \%$ of patients. Detailed reporting of all relevant neurologic outcomes would provide additional insight into the benefits of remaining in the cerebral autoregulation zone. In addition, according to multivariable regression modeling, the optimal MAP may be higher for nonwhite patients and lower for patients receiving diuretics, those with previous carotid endarterectomy and lower per 
every 60 minutes of time on CPB. However, the $R^{2}$ was only 0.1002 in this model, suggesting that these predictors explained only $10 \%$ of the variation in MAP observed among patients. Thus, although one may consider adjusting MAP based on these demographic and operative factors, whether clinical outcomes will be improved remains to be seen. Although TCD has a clinical role outside of the operating room, it remains primarily a research tool in cardiac surgery operating rooms. The authors suggested that near-infrared spectroscopy may be a more clinically relevant tool for practice $^{8}$; based on our personal experience, it seems much easier to use than TCD, at least for nonexperts.

Again, we congratulate the authors on their perseverance in tackling such an important issue in cardiac surgery using sophisticated and validated tools to measure CBF. A randomized clinical trial comparing the standard of care to MAP management based on cerebral autoregulation as measured by near-infrared spectroscopy and TCD is ongoing (ClinicalTrials.gov: NCT00981474). The primary outcome is appropriate; a composite of clinical neurologic changes and new ischemic lesions detected on magnetic resonance imaging during the perioperative period. We eagerly await the results of this clinical trial. In the meantime, intraoperative blood pressure management can be guided by the authors' results. Hitting the "sweet spot" for cerebral autoregulation during CPB remains elusive.

\section{References}

1. Gottesman RF, Sherman PM, Grega MA, Yousem DM, Borowicz LM, Selnes OA, et al. Watershed strokes after cardiac surgery: diagnosis, etiology, and outcome. Stroke. 2006;37:2306-11.

2. Hori D, Brown M, Ono T, Rappold T, Sieber F, Gottschalk A, et al. Arterial pressure above the upper cerebral autoregulation limit during cardiopulmonary bypass is associated with postoperative delirium. Br J Anaesth. 2014;113: 1009-17.

3. Ono M, Brady K, Easley RB, Brown C, Kraut M, Gottesman RF, et al. Duration and magnitude of blood pressure below cerebral autoregulation threshold during cardiopulmonary bypass is associated with major morbidity and operative mortality. J Thorac Cardiovasc Surg. 2014;147:483-9.

4. Ono M, Joshi B, Brady KM, Easley RB, Zheng Y, Brown C, et al. Risks for impaired cerebral autoregulation during cardiopulmonary bypass and postoperative stroke. Br J Anaesth. 2012;109:391-8.

5. Hori D, Nomura Y, Ono M, Joshi B, Mandal K, Cameron D, et al. Optimal blood pressure during cardiopulmonary bypass defined by cerebral autoregulation monitoring. J Thorac Cardiovasc Surg. 2017;154:1590-8.

6. Czosnyka M, Brady K, Reinhard M, Smielewski P, Steiner LA. Monitoring of cerebrovascular autoregulation: facts, myths, and missing links. Neurocrit Care. 2009; 10:373-86.

7. Steiner LA, Coles JP, Johnston AJ, Chatfield DA, Smielewski P, Fryer TD, et al Assessment of cerebrovascular autoregulation in head-injured patients: a validation study. Stroke. 2003;34:2404-9.

8. Brady K, Joshi B, Zweifel C, Smielewski P, Czosnyka M, Easley RB, et al Real-time continuous monitoring of cerebral blood flow autoregulation using near-infrared spectroscopy in patients undergoing cardiopulmonary bypass. Stroke. 2010;41:1951-6. 